\title{
3D composite particles
}

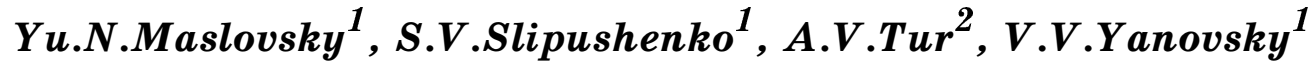 \\ ${ }^{1}$ Institute for Single Crystals, STC "Institute for Single Crystals" National \\ Academy of Sciences of Ukraine, 60, Lenin ave. 61001 Kharkiv, Ukraine \\ ${ }^{2}$ Universite de Toulouse [UPS], CNRS, Institut de Recherche en \\ Astrophysique et Planetologie, 9 avenue du Colonel Roche, BP 44346, \\ 31028 Toulouse Cedex 4, France
}

\section{Received November 10, 2014}

The simple model of three-dimensional particle with internal degrees of freedom that interact with each other is proposed. The typical kinematic modes of motion of such particle with a small number of internal degrees of freedom are considered. Analytically investigated are all modes of motion of particle with one internal degree of freedom. We show that appearance of chaotic regimes is typical and found the mechanism of chaotization starting from two internal degrees of freedom. Obtained are the basic rules of motion of such particles and of the redistribution of energy of the internal degrees of freedom.

Keywords: composite particles, internal degrees of freedom, kinematic modes, billiard formalism, distribution of energy.

Предложена простая модель трехмерной частицы с внутренними степенями свободы, которые взаимодействуют друг с другом. Рассмотрены типичные кинематические режимы движения такой частицы с небольшим числом внутренних степеней свободы. Аналитически исследованы все режимы движения при одной внутренней степенью свободы. Показано типичность появления хаотических режимов и установлен механизм хаотизации уже при двух внутренних степенях свободы. Получены основные закономерности движения таких частиц и закономерности перераспределение энергии по внутренним степеням свободы.

3D композитні частинки. Ю.М.Масловсъкий, С.В.Сліпушенко, А.В.Тур, В.В.Яновсъкий

Запропоновано просту модель тривимірної частинки із внутрішніми ступенями свободи, які взаємодіють одна з одною. Розглянуто типові кінематичні режими руху такої частки $з$ невеликою кількістю внутрішніх ступенів свободи. Аналітично досліджені всі режими руху з одною внутрішньою ступеню свободи. Доведено типовість появи хаотичних режимів та встановлено механізм хаотизации вже при двох внутрішніх ступенях свободи. Отримано основні закономірності руху таких частинок та закономірності перерозподіл енергії по внутрішніх ступенях свободи.

\section{Introduction}

The interest in the nanoobjects having a small number of internal degrees of freedom has grown recently. Simple one-dimensional models have been proposed in paper [1]. In this work also were found anomalous laws of reflection of such objects from perfectly elastic barriers. Anomalous properties also appear in their motion in external fields [2]. Even more interesting properties arise when these objects collide with each other [3]. The transition to more realistic models involves raising of the dimension of such objects up to three-dimensional. It should be noted, in principle, that preservation of discovered 
effects in this case could be expected. Indeed, numerical simulation of collisions of argon nanoparticles with structureless wall discovered the preservation of abnormal laws [4].

In the paper we propose a three-dimensional model of particles with a small number of internal degrees of freedom. The billiard formalism is generalized to this case. It is shown that the kinematic modes of motion of such objects can be studied using a multi-dimensional billiards with one point-like moving particle. Analytically investigated are all modes of motion of particles with one internal degree of freedom. It is shown that appearance of chaotic regimes is typical already for two internal degrees of freedom. Predicted are the laws of wandering of such freely moving particle under the influence of internal degrees of freedom and the redistribution of energy over the degrees of freedom.

\section{Three-dimensional composite particles}

In this section we introduce more general composite particles than were considered in papers [1], [2], [3]. Let a spherical shell contain $N$ balls, which can elastically collide with each other and the shell (see Fig.1). Particles, enclosed in a shell play the role of internal degrees of freedom. For simplicity, let us assume that a spherical shell of radius $R_{0}$ and mass $m_{0}$ has zero thickness. Internal particle of radii $R_{i}<R_{0}$ have mass $m_{i}$. The index $i=1, \ldots, N$ numbers internal particles. Let's discuss the possibility of description of kinematics and dynamics of such composite particles via billiard approach [5], [1]. In this approach, the motion of composite particles and their collisions are determined by the motion of one point particle in multidimensional billiard of special form.

A complete description of positions of all constituting the composite particle elements is achieved by determining the coordinates of the shell center $\vec{r}_{0}$ and the centers of all internal balls $\vec{r}_{i}$. It is convenient to introduce a common notation for all of these vectors

$$
\vec{r}=\left(\vec{r}_{0}, \vec{r}_{1}, \ldots, \vec{r}_{N}\right) \text {. }
$$

Of course, each element of the composite particles can move with some speed. So the speed of the shell we will denote as $\dot{\vec{r}}_{0}$, and the velocities of the internal particles as $\dot{\vec{r}}_{i}$. It is also natural to introduce a common notation for all these velocities as

$$
\vec{u}=\left(\dot{\vec{r}}_{0}, \dot{\vec{r}}_{1}, \ldots, \dot{\vec{r}}_{N}\right)
$$

Thus the position of all elements of the composite particle is described by a point in Euclidean space $\mathcal{R}^{3(N+1)}$. However, not every point $\mathcal{R}^{3(N+1)}$ corresponds to a possible position of the elements of the composite particle. So let's discuss its configuration space. The configuration space is the space of all possible positions of the elements of the composite particle. The main limitation of the configuration space connected with the impossibility of penetration of solid balls into each other and throughout the shell of composite particle. The condition of the impossibility of $i$-th internal particle to penetrate into the $j$-th inner particle is written as [5]

$$
\left(\vec{r}_{i}-\vec{r}_{j}\right)^{2} \geq\left(R_{i}+R_{j}\right)^{2}, \quad i, j=1, \ldots, N .
$$

The number of such conditions, corresponding to impossibility of the interpenetration of all pairs of inner particles is $N(N-1) / 2$. Each such inequality determines a volume belonging to the configuration space $\mathcal{R}^{3(N+1)}$. Equality defines an elliptic cylinder with a two-dimensional basis in the form of sphere $S^{2}$ (see for example [8]). Physically this equality defines the boundary of the configuration space, which corresponds to the collision of a certain pair of internal particles. Thus each inequality defines the outer part of certain elliptic cylinder in $\mathcal{R}^{3(N+1)}$. All these inequalities define the part of $\mathcal{R}^{3(N+1)}$ corresponding to the intersection of all externals of the $N(N-1) / 2$ cylinders.

Conditions prohibiting the exit of the internal particles outside the shell are of the form

$$
\left(\vec{r}_{0}-\vec{r}_{i}\right)^{2} \leq\left(R_{0}-R_{i}\right)^{2}, \quad i=1 \ldots N
$$




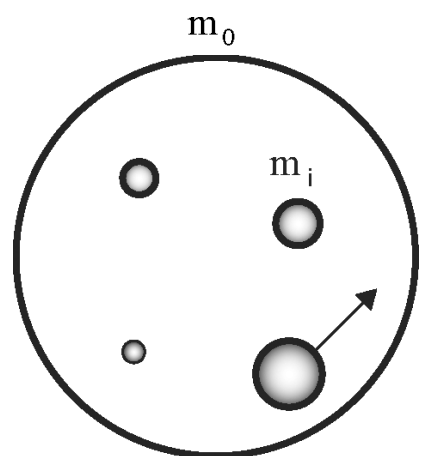

Fig. 1. The cross section of the composite particle with multiple internal particles of various radii and masses is shown.

and each such condition defines an interior of an elliptical cylinder with the base in a form of a sphere $S^{2}$ in space $\mathcal{R}^{3(N+1)}$. Equality defines an elliptic cylinder, which corresponds to the collision of certain internal particle with the shell. Number of such conditions is $N$. Thus, the configuration space of composite particle is determined by the intersection of the exterior of $N(N-1) / 2$ cylinders and the interior of $N$ cylinders.

Let's consider now the motion of elements constituting the composite particle. Before the collision between any of its elements, their coordinates are changing according to the equation

$$
\vec{r}(t)=\left.\vec{r}\right|_{t=0}+\vec{u} t
$$

in this equation it is easy to recognize a parametrically given rectilinear trajectory of a point particle in $3(N+1)$-dimensional configuration space. At the moment of collision between pair of elements constituting composite particle, this line reaches a certain boundary in the configuration space. Next, having the velocities of these elements after collision determined from the conservation laws, we can continue the trajectory. Such continuation will appear in the configuration space as a reflection from its boundary. It remains to check the validity of usual for billiards law of reflection. At billiards reflection the tangential velocity at the point of reflection is saved, while the normal changes its sign. It is easy to understand that in the considered case it is not true. To enforce the fulfillment of such law of reflection following [5] we transfer to the billiard coordinates $\vec{\rho}_{i}=\sqrt{m_{i}} \vec{r}_{i}$ or

$$
\vec{\rho}=\left(\sqrt{m_{0}} \vec{r}_{0}, \sqrt{m_{1}} \vec{r}_{1}, \ldots, \sqrt{m_{N}} \vec{r}_{N}\right)
$$

The vector velocities in the billiard coordinates are

$$
\vec{v}=\left(\sqrt{m_{0}} \dot{\vec{r}}_{0}, \sqrt{m_{1}} \dot{\vec{r}}_{1}, \ldots, \sqrt{m_{N}} \dot{\vec{r}}_{N}\right)
$$

One of the advantages of the new coordinates is that the length of a velocity vector $\vec{v}=\dot{\vec{\rho}}$ is determined by the total kinetic energy of the composite particle:

$$
|\vec{v}|=\sqrt{\sum_{i=0}^{N+1} m_{i} \dot{\vec{r}}_{i}^{2}}=\sqrt{2 E}
$$

Accordingly, the law of conservation of energy guaranties the conservation of the length of the velocity vector in the process of reflection of the point particle from the boundary of the configuration space. The second necessary condition of specular reflection is the change of sign of the normal velocity component. For the borders of the configuration space corresponding to the internal collision of particles, justice of the billiards law of reflection was proved in [5]. Therefore, we only need to verify the fulfillment of the law of reflection for the boundaries in the configuration space, corresponding to the collision of intemal particles with the shell of composite particle. 


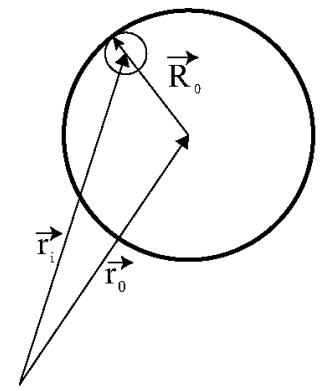

Fig. 2. Show the corresponding vectors at the moment of collision of the shell and $i$-th inner particle. It is important to note that the touch point and spheres centers lie on the same line.

Let's consider the collision of the shell with the $i$-th internal particle. Let the trajectory of the point effective particle reach the boundary (2) in the configuration space at the point $\vec{\rho}$. Of course, equation (2) should be recorded in the billiards coordinates. Let's calculate the normal to the boundary at this point. The vector directed along the normal to this surface is easy to find

$$
\vec{\sigma}=\left(\frac{2}{\sqrt{m_{0}}}\left(\frac{\vec{\rho}_{0}}{\sqrt{m_{0}}}-\frac{\vec{\rho}_{i}}{\sqrt{m_{i}}}\right), 0 \ldots, 0,-\frac{2}{\sqrt{m_{i}}}\left(\frac{\vec{\rho}_{0}}{\sqrt{m_{0}}}-\frac{\vec{\rho}_{i}}{\sqrt{m_{i}}}\right), 0 \ldots, 0\right)
$$

normalizing its length we will receive the unit vector, normal to the boundary at the point $\vec{\rho}$

$$
\vec{n}=\frac{1}{\left|R_{0}-R_{i}\right| \sqrt{m_{0}+m_{i}}}\left(\sqrt{m_{i}}\left(\frac{\vec{\rho}_{0}}{\sqrt{m_{0}}}-\frac{\vec{\rho}_{i}}{\sqrt{m_{i}}}\right), 0 \ldots, 0,-\sqrt{m_{0}}\left(\frac{\vec{\rho}_{0}}{\sqrt{m_{0}}}-\frac{\vec{\rho}_{i}}{\sqrt{m_{i}}}\right), 0 \ldots, 0\right) .
$$

At the point of collision of trajectory with the boundary vectors $\frac{\vec{\rho}_{0}}{\sqrt{m_{0}}}$ and $\frac{\vec{\rho}_{i}}{\sqrt{m_{i}}}$ are connected in a certain way, see Fig.2. In other words $\frac{\vec{\rho}_{i}}{\sqrt{m_{i}}}-\frac{\vec{\rho}_{0}}{\sqrt{m_{0}}}=\vec{R}_{0}-\vec{R}_{i}$, and direction of the vector $\vec{R}_{i}$ coincides with the direction of the vector $\vec{R}_{0}$. Vector $\vec{R}_{0}$ connects the point of spheres contact with the shells center. This allows to record a normal as

$$
\vec{n}=\frac{1}{\left|R_{0}-R_{i}\right| \sqrt{m_{0}+m_{i}}}\left(-\sqrt{m_{i}}\left(\vec{R}_{0}-\vec{R}_{i}\right), 0 \ldots, 0, \sqrt{m_{0}}\left(\vec{R}_{0}-\vec{R}_{i}\right), 0 \ldots, 0\right) .
$$

Taking into account that the length of the vector $\vec{v}$ is saved, to verify the billiards law of reflection it is enough to ensure that reflection from a point $\vec{\rho}$ satisfies following equations:

$$
\vec{v}_{\perp}=-\vec{v}_{\perp}^{\prime} .
$$

In coordinate form, this law can be written as

$$
\vec{v} \cdot \vec{n}=\frac{\sqrt{m_{0} m_{i}}}{\left|R_{0}-R_{i}\right| \sqrt{m_{0}+m_{i}}}\left(\vec{u}_{i}-\vec{u}_{0}\right) \cdot\left(\vec{R}_{0}-\vec{R}_{i}\right) .
$$

The collision between a shell and inner particle is perfectly elastic, therefore, Newton's law of collisions is satisfied. For colliding spherical particles this means that the projection of the velocity difference $\vec{u}_{i}-\vec{u}_{0}$ on the direction connecting the centers of particles changes the sign. Accordingly, the normal component of the velocity of the billiard's particle changes its sign in the process of reflection from the boundary of the configuration billiard. Similarly can be proved the justice of the billiard's law of reflection from the boundary in the configuration space corresponding to the collision of any two components of composite particle. Thus it is proved that the movement of composite particle can be described as the efficient movement of particle in multi-dimensional billiard. The form of this billiard is determined by the size of the individual components of the composite particle and in general is the intersection of $N(N+1)$ cylinders. The boundary of the billiard contains both outer and inner cylinder walls, therefore it has areas with positive and negative curvature. This suggests the existence of chaotic regimes of motion of complex composite particles. However, in the simplest case of one internal particle, the problem is significantly simplified. Let's consider this case in detail. 


\section{Composite particle with one degree of freedom}

Let's consider the kinetic regime of motion of the composite particle with one internal degree of freedom. As discussed above, such system can be reduced to a billiard in six-dimensional space. Due to the presence of only one internal particle boundaries of the configuration space are defined by one condition of impossibility of escaping the shell

$$
\left(\frac{\vec{\rho}_{0}}{\sqrt{m_{0}}}-\frac{\vec{\rho}_{1}}{\sqrt{m_{1}}}\right)^{2}=\left(R_{0}-R_{1}\right)^{2}
$$

here $R_{0}$ is the shell radius and $R_{1}$ is the radius of the inner particle. As discussed above this is a cylinder with base $\mathcal{S}^{2}$ in six-dimensional space $\left(\vec{\rho}_{0}, \vec{\rho}_{1}\right) \in \mathcal{R}^{6}$. Note that a composite particle itself is a closed system. And therefore its center of mass moves with constant velocity. The speed of this movement is determined by the total momentum divided by the total mass of the shell and internal particle $\vec{V}_{c}=\left(\sqrt{m_{0}} \vec{v}_{0}+\sqrt{m_{1}} \vec{v}_{1}\right) /\left(m_{0}+m_{1}\right)$. In other words, position of the center of mass changes according to the equation

$$
\sqrt{m_{0}} \vec{\rho}_{0}+\sqrt{m_{1}} \vec{\rho}_{1}=\left(t \vec{V}_{c}+\vec{r}_{c}\right)\left(m_{0}+m_{1}\right) ;
$$

here $\vec{r}_{c}$ is the initial position of the center of mass at $t=0$. From a geometrical point of view, these relations define a three-dimensional hyperplane in the configuration space

$$
\begin{aligned}
& \sqrt{m_{0}} \rho_{01}+\sqrt{m_{1}} \rho_{11}=t V_{c 1}+r_{c 1} ; \\
& \sqrt{m_{0}} \rho_{02}+\sqrt{m_{1}} \rho_{12}=t V_{c 2}+r_{c 2} ; \\
& \sqrt{m_{0}} \rho_{03}+\sqrt{m_{1}} \rho_{13}=t V_{c 3}+r_{c 3} .
\end{aligned}
$$

The area of intersection of this hyperplane with the cylindrical billiard in the configuration space is easy to find. Projecting the billiard trajectory on this intersection we transfer to the motion of point particle in three-dimensional billiard. The law of reflection of projection of the trajectory from the borders of this billiard preserves specular character. Therefore, it is sufficient to study the movement of efficient particle in a billiard of this section. The equation defining the boundary of a section is easy to find

$$
\left(\vec{\rho}_{0}-\sqrt{m_{0}}\left(t \vec{V}_{c}+\vec{r}_{c}\right)\right)^{2}=\frac{m_{0} m_{1}^{2}\left(R_{0}-R_{1}\right)^{2}}{\left(m_{0}+m_{1}\right)^{2}} .
$$

It is easy to recognize a sphere $\mathcal{S}^{2}$ in three-dimensional space $\mathcal{R}^{3} \ni \vec{\rho}_{0}$ in this equation. The center of this sphere is at point $\vec{\rho}_{0}=\sqrt{m_{0}}\left(t \vec{V}_{c}+\vec{r}_{c}\right)$. Taking into account that the three-dimensional hypersurface with time shifts in a parallel manner, as a convenient section we may choose a hyperplane passing through the origin and project billiard trajectory on the cross section of the cylinder by this hyperplane. Thus, the effective point particle moves in a spherical billiard of radii $R_{e f}=\frac{\sqrt{m_{0}} m_{1}\left|R_{0}-R_{1}\right|}{m_{0}+m_{1}}$. Properties of the trajectories in such billiard are defined by a simple fact. The velocity vectors of the incident and reflected trajectory lie in the plane passing through the center of the sphere. This means that the motion of a billiard trajectory occurs only in this plane. Then it is enough to consider the movement of efficient particle in this plane. This simplifies the shape of the billiard. Billiard arising in the cross-section of the sphere coincides with the billiard in a circle with radius $R_{e f}$ (see Fig.3). The type of trajectories in the circle is well studied (see for example [6]). Everything is determined by the angle $\varphi$ shown at Fig.3. If this angle is commensurate with $\pi$ the trajectory is periodic. So in case of $\varphi=2 \pi \frac{n}{m}$ ( $m$ and $n$ are irreducible integers) the trajectory is $m$-periodic and makes $n$ turns around a circle. The number of rotations of the trajectory is $\mu=\frac{n}{m}$. In the case of incommensurability of $\varphi$ with $\pi$ the points of reflection of the trajectory fill the circle everywhere densely. Thus there can exist only two types of lateral movement, periodic with any period and quasi-periodic. Combining them with a constant longitudinal movement leads to a complete description of the types of effective point particles trajectories in the original billiard 


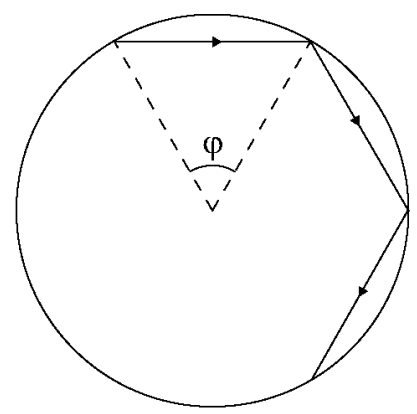

Fig. 3. Billiard in cross section by a hyperplane passing through the coordinates origin and the initial segment of the trajectory of transverse movements. Three segments of the billiard's trajectory are shown.

and hence to the description of all possible regimes of motion of the composite particle with one internal degree of freedom. Such simple structure of the kinetic regime of composite particle is due to the fact that the evolution of the particle contains five integrals of motion in involution. These are three projections of the total momentum, projection of the total angular momentum on the plane of motion and the total energy of the composite particle.

Let us now consider how the efficient movement of billiard particle is interpreted in terms of the movement of the individual components of the composite particle. It is easy to notice that the projection of the trajectory of effective particle in the billiard (4) has a simple physical meaning of the movement of the shell. In other words, the shell of composite particle moves as if it is mirrored from the walls of the circular billiard with radius

$$
R_{s}=\frac{m_{1}\left|R_{0}-R_{1}\right|}{m_{0}+m_{1}}
$$

The center of this billiard moves according to the law that corresponds to the movement of the center of mass of the composite particle $t \vec{V}_{c}+\vec{r}_{c}$. The trajectory in the circular billiard is a broken line, each segment of which is rotated relative to the previous one on the angle $\varphi$ (Fig.3). This angle is determined by the initial conditions

$$
\varphi=2 \arccos \frac{\sqrt{\frac{\left(\left(\vec{r}_{0}-\vec{r}_{c}\right)\left(\vec{u}_{0}-\vec{V}_{c}\right)\right)^{2}}{\left(\vec{u}_{0}-\vec{V}_{c}\right)^{2}}+R_{s}^{2}-\left(\vec{r}_{0}-\vec{r}_{c}\right)^{2}}}{\left|\left(\vec{r}_{0}-\vec{r}_{c}\right)+\left(\sqrt{\frac{\left(\left(\vec{r}_{0}-\vec{r}_{c}\right)\left(\vec{u}_{0}-\vec{V}_{c}\right)\right)^{2}}{\left(\vec{u}_{0}-\vec{V}_{c}\right)^{2}}+R_{s}^{2}-\left(\vec{r}_{0}-\vec{r}_{c}\right)^{2}}-\frac{\left(\vec{r}_{0}-\vec{r}_{c}\right)\left(\vec{u}_{0}-\vec{V}_{c}\right)}{\left|\vec{u}_{0}-\vec{V}_{c}\right|}\right) \frac{\vec{u}_{0}-\vec{V}_{c}}{\left|\vec{u}_{0}-\vec{V}_{c}\right|}\right|},
$$

here $\vec{r}_{0}$ and $\vec{u}_{0}$ denote the position and velocity of the shell at the initial moment of time $t=0$. The magnitude of this angle determines whether the trajectory is periodic or quasiperiodic. Also, it is easy to estimate the time needed for a shell to make a full rotation around the center of mass of the composite particle

$$
T \simeq \frac{2 \pi}{\varphi} \tau_{s}
$$

where $\tau_{s}$ is the time between individual collisions of shell with the inner particle. Naturally, this relation is exact only for trajectories closing after one turn around the center of mass. The value $\tau_{s}$ is easily determined due to the property of preservation of the value of velocity in the billiard

$$
\tau_{s}=\frac{2 R_{s} \sin \frac{\varphi}{2}}{\left|\vec{u}_{0}\right|} .
$$

Thus, the shell movement is a broken spiral with radius $R_{s}$. Its axis directed along $\vec{V}_{c}$. The travel time for one individual segment of the spiral is $\tau_{s}$, the duration of full turn is $T$. The step of such spiral is equal to $s=T V_{c}$. In the composite particle with one degree of freedom, the position of the internal particle is 
directly related to the position of the shell $m_{0}\left(\vec{r}_{0}-t \vec{V}_{c}-\vec{r}_{c}\right)=-m_{1}\left(\vec{r}_{1}-t \vec{V}_{c}-\vec{r}_{c}\right)$ (see equation (3)). Accordingly, the internal particle in kinematic regime also moves in a similar broken spiral, but with different radius $R_{i}=\frac{m_{0}\left|R_{0}-R_{1}\right|}{m_{0}+m_{1}}$.

Let's consider oscillations of the shell in the kinematic regime of motion of the composite particle. Shell of the composite particle makes a circular motion of radius $R_{s}$ around the center of inertia in the plane with normal

$$
\vec{N}=\frac{\left(\vec{r}_{0}-\vec{r}_{c}\right) \times\left(\vec{u}_{0}-\vec{V}_{c}\right)}{\left|\left(\vec{r}_{0}-\vec{r}_{c}\right) \times\left(\vec{u}_{0}-\vec{V}_{c}\right)\right|}
$$

The axis of the spiral makes a certain angle $\psi$ with the normal to the plane in which the shell $<<$ rotates $>>$ around the center of inertia. Its value

$$
\psi=\arccos \frac{\vec{V}_{c} \vec{N}}{\left|\vec{V}_{c}\right||\vec{N}|} .
$$

Accordingly, in the projection on the direction of movement of the center of mass the amplitude of longitudinal oscillations will be

$$
a_{l}=2 R_{s} \sin \psi .
$$

In the plane transverse to the direction of motion the shell will move along a broken line inscribed in the ellipse. The major semiaxis of the ellipse is directed along the direction $\vec{V}_{c} \times \vec{N}$. The amplitude of the transverse oscillations in this direction is equal $a_{t \max }=2 R_{s}$. In the orthogonal direction, the amplitude of the transverse oscillations is equal $a_{t \min }=2 R_{s} \cos \psi$. Naturally, the frequencies of these oscillations are identical and equal to $1 / T$. Recall that these oscillations are not sinusoidal, they are composed of individual sites of rectilinear motion. Each such site has a duration $\tau_{s}$.

Let's consider another important aspect of the motion of the composite particle, the distribution of energy between internal degree of freedom and shell. Relative coordinates of internal particle and shell are rigidly connected: $m_{0}\left(\vec{r}_{0}-t \vec{V}_{c}-\vec{r}_{c}\right)=-m_{1}\left(\vec{r}_{1}-t \vec{V}_{c}-\vec{r}_{c}\right)$. Respectively, their relative speeds are also connected: $m_{0}\left(\vec{u}_{0}-\vec{V}_{c}\right)=-m_{1}\left(\vec{u}_{1}-\vec{V}_{c}\right)$. Since both the shell and internal particle move following the billiard's law relative to the center of inertia, the magnitudes of their relative velocities will be time preserved. Accordingly, the ratio of their relative motion kinetic energies is very simple

$$
\frac{E_{0}}{E_{1}}=\frac{m_{1}}{m_{0}}
$$

From this relation it follows that in the case of light internal particle and heavy shell, almost all the kinetic energy of the relative motion will be concentrated in the internal degree of freedom. Thus we have completely described the characteristics of the kinetic regime of motion of the composite particle.

Now let us consider which of these characteristics can be determined in the experiment. Naturally, we can observe only a shell of composite particle. It is of interest therefore the question of determining of the internal state of composite particle from the observed data. As discussed above, the shell of composite particle oscillates in some plane around the center of inertia, which moves uniformly and rectilinearly. Accordingly, observing the shell for a long time and averaging its position we can determine the speed of movement of the center of mass of the composite particle $\vec{V}_{c}$. This makes possible the determination of the relative velocity of the shell $\vec{u}_{0}-\vec{V}_{c}$, and the direction of the relative movement of the internal particle, because it is opposite to the direction of the relative velocity of the shell. Also it is possible to define the normal vector to the plane in which the shell performs oscillatory motion $\vec{N}$. In this plane the shell and internal particle move along broken line inscribed in a circle. In the experiment it is possible to determine the radius of this circle for the shell $R_{s}$. If from indirect data we can assume that the size of inner freedom $R_{1}$ is small compared to the size of the shell $R_{0}$, it is easy to obtain mass estimates for the internal particle and shell

$$
m_{0} \simeq\left(1-\frac{R_{s}}{R_{n}}\right) M \quad m_{1} \simeq \frac{R_{s}}{R_{0}} M
$$


where $M$ is a mass of composite particle. In this case, using the relationship between the location of the shell and interior particle relative to the center of mass, from the position of the shell it is easy to determine the position of the inner particle. In other words, by observing the movement of the shell of composite particle with internal degree of a small mass, it is possible to get the properties of the internal degree of freedom.

\section{Composite particle with two or more degrees of freedom}

We now transfer to the discussion of the behavior of composite particle with two internal degrees of freedom. First of all it is necessary to discuss the structure of billiard in which the efficient point particle moves. The boundary of this billiard in 9-dimensional space $\vec{\rho} \in R^{9}$ is determined by three conditions. One condition is impossibility of mutual penetration of the internal particles during collision

$$
\left(\frac{\vec{\rho}_{1}}{\sqrt{m_{1}}}-\frac{\vec{\rho}_{2}}{\sqrt{m_{2}}}\right)^{2}=\left(R_{1}+R_{2}\right)^{2}
$$

which defines the part of boundary of the billiard with a concave or scattering surface. More precisely it is semi scattering billiard [7], with areas with a curvature different from zero only in two directions. This boundary corresponds to the part of the elliptical cylinder with 2-basement (basis of two-dimensional ellipsoid) [8]. The configuration space is contained in the outer part of this cylinder. Two other conditions corresponding to the impossibility for internal particles to leave the shell have the form

$$
\begin{gathered}
\left(\frac{\vec{\rho}_{0}}{\sqrt{m_{0}}}-\frac{\vec{\rho}_{1}}{m_{1}}\right)^{2}=\left(R_{0}+R_{1}\right)^{2} ; \\
\left(\frac{\vec{\rho}_{0}}{\sqrt{m_{0}}}-\frac{\overrightarrow{\rho_{2}}}{\sqrt{m_{2}}}\right)^{2}=\left(R_{0}+R_{2}\right)^{2} .
\end{gathered}
$$

These conditions determine the convex or focusing part of the boundary of the billiard. The same way as above, the border is a part of the intersection of two elliptic cylinders with 2-basements. The configuration space is the inner part of the intersection of these cylinders. Thus the movement of efficient point particle occurs in the billiard, having generally both scattering and focusing sites of the border. According to modern concepts the motion of a particle in such billiard is chaotic [9], [10]. It should be noted that the relative arrangement of these cylinders plays an important role in the classification of possible regimes of motion. In particular, it is important to check the possibility of such arrangement of cylinders that the boundary of the billiard contains only scattering sites. It is easy to understand that this case may not be realized. Also may not be realized the case of billiard with focusing only border. The condition of consistency of the system of equations above is always satisfied. Thus the movement of efficient particle occurs in a billiard with focusing and scattering boundaries. However, it should be noted that such a multi-dimensional billiard is an open billiard. There are directions, moving along which a point particle will never meet the border of billiard. All directions with this property are spanned by three orthogonal vectors $\vec{n}_{1}=(1,0,0,1,0,0,1,0,0), \vec{n}_{2}=(0,1,0,0,1,0,0,1,0), \vec{n}_{3}=(0,0,1,0,0,1,0,0,1)\left(\vec{n}_{i} \cdot \vec{n}_{j}=0\right.$ if $i \neq j, i, j=1,2,3)$. Accordingly, the trajectories moving in directions $\vec{k}=a \vec{n}_{1}+b \vec{n}_{2}+c \vec{n}_{3}$ never meet the border of billiard. Here $a, b$ and $c$ are arbitrary numbers. Dynamics of particle along these directions is trivial. This means that such subspace we may left unconsidered and focus on 6-dimensional space of orthogonal directions.

Another interesting consequence of the analysis of billiard's structure concerns billiards with several particles, which may encounter collisions during their movement inside billiard. Such billiards recently been considered in [11]. It is interesting to note that a billiard in circle with two colliding disks inside is reduced to a multidimensional normal billiard with a single effective point particle in a special billiard described above. This means that some properties of the regular billiard are relevant for the billiard with collisions between particles. In particular, a gas of colliding particles in a spherical vessel corresponds to the considered above composite particle with infinite mass of shell. 
Returning to the kinematics of composite particle from general reasons it is clear that this billiard implements movement of chaotic trajectories with positive Lyapunov exponent. In other words, it implements strong chaos. Of course, there are a countable number of periodic trajectories in the phase space. Due to the multidimensional nature of the phase space, computation of the Lyapunov exponent and visual demonstration of chaotic behavior is complicated. However, from the physical point of view it is interesting to find out the behavior of the shell of such structurally complex particle. The reason for this is that the shell behavior is observable. This means that main attention should be focused on the degrees of freedom associated with the shell.

So let's discuss the deviation of the shell from the position of the center of mass at the collision with the inner particles. To estimate the deviation of the shell with $N$ internal degrees of freedom let us assume, for simplicity, that their masses are close to some characteristic mass $m_{i} \approx m$, and the sizes of the internal particles $R_{i} \approx R$. Then passing to a reference system with the centre of inertia at the origin, we consider the characteristic displacement of the shell due to collisions with the internal particles. It is easy to prove that the deviation of the shell from the position of the centre of inertia is determined by the ratio

$$
\Delta \vec{r}_{0}=-\frac{m}{m_{0}+N m} \sum_{i}^{N} \Delta \vec{r}_{i},
$$

where $\Delta \vec{r}_{i}$ is the vector distance between $i$-th inner particle and the shell. The length of this vector is bounded above by the value $\left(R_{0}-R\right)$ in the reference frame of the center of inertia. Then it is easy to estimate the standard deviation of the shell as

$$
\Delta r_{0} \approx\left(R_{0}-R\right) \sqrt{\frac{m^{2} N}{\left(m_{0}+N m\right)^{2}}} .
$$

At the derivation of this relation we use the assumption of randomness of the directions of the placement of internal particles and assumption of a zero mean vector of the placement of internal particles in the system center of mass. It is easy to see that for one internal degree of freedom this ratio coincides with the previously obtained relation (5). In addition, it is important to note that the magnitude of the deviation decreases with increase of a number of internal degrees of freedom $\Delta r_{0} \sim 1 / \sqrt{N}$ and therefore for macroscopic bodies such deviation disappears. This effect should be observed only for particles with a small number of internal degrees of freedom.

Of course, the behavior of displacement with time, due to the chaotic motion of such particle would have in the general case the nature of random chaotic walks (when $N \geq 2$ ). This unusual process of shell walks occurs due to the impacts of internal particles with the shell. It should be noted that in this movement the position of the center of inertia of the system is always located inside the shell. The presence of chaotic motion is an important difference from the case of periodic motion of a particle with one internal degree of freedom (see section 3). It should be noted that in the degenerate cases it is also possible to be realized for periodic modes of motion of the shell having larger number of internal degrees of freedom. The presence of periodic regimes is a consequence of the existence of a countable number of unstable orbits in the chaotic region of the phase space. As a simple example of two-periodic trajectory for a particle with two internal degrees of freedom $m_{1}=m_{2}$ and $R_{1}=R_{2}$ we may refer to a trajectory which before the collision with the boundary moves along the direction $\vec{u}=\left(\vec{u}_{0}=0, \vec{u}_{1},-\vec{u}_{1}\right)$ for a time $T=\frac{R_{0}-2 R_{1}}{u_{1}}$, then it is reflected in the direction $\vec{u}=\left(\vec{u}_{0}=0,-\vec{u}_{1}, \vec{u}_{1}\right)$ and moves again during the time $T=\frac{R_{0}-2 R_{1}}{u_{1}}$ until next collision with the boundary. Then the cycle repeats. The period of such trajectory is equal to $2 T$. For this trajectory, the initial positions of the internal particles are selected symmetrically with respect to a shell.

Let's consider now the distribution of energy over the degrees of freedom. Recall that in the center of inertia the total momentum of the shell and internal particle is zero. Accordingly the following equation is satisfied

$$
m_{0} v_{0}=-\sum_{i=1}^{N} m_{i} \vec{v}_{i} \equiv M_{i n} V_{i n}
$$


here $M_{i n}$ is the total mass of the internal degrees of freedom, and $V_{i n}$ is the velocity of the centre of inertia only of internal degrees of freedom. Using this relation we will receive the ratio of the energy of the shell and internal degrees of freedom in the centre of inertia

$$
\frac{E_{0}}{E_{i n}}=\frac{m}{m_{0}}
$$

where $E_{0}$ is the energy of the shell, and $E_{i n}$ is the energy of the internal degrees of freedom in the system of reference of the centre of mass, $m$ - is the mass of internal degree of freedom. This relation coincides with the previously obtained equation (6) in the case of one degree of freedom. Assuming even distribution of the energy over the internal degrees of freedom, from this relation it is easy to obtain the ratio of the shell's energy to the energy $E$ of one internal particle

$$
\frac{E_{0}}{E}=\frac{M_{i n}}{m_{0}} .
$$

Of course, these relations are satisfied in the frame of reference of the centre of inertia of the entire system. Numerical simulation of the behavior of composite particles with many internal degrees of freedom is in good agreement with the retrieved relations.

\section{Conclusions}

Thus the description of the properties of particles with internal degrees of freedom is quite simple in the proposed billiard's approach. This approach is also natural to use for more complex problems, for example, to study of the laws of collision of particles with internal degrees of freedom. Naturally we should expect qualitative changes in the scattering of such particles in comparison with macroscopic particles, both structureless and with a large number of internal degrees of freedom. The received in the paper general properties should be observed in any realistic particles with internal degrees of freedom. Important element of dynamics of such particles is the redistribution of the energy over the internal degrees of freedom and typical regimes of their movement.

\section{References}

1. V.V.Yanovsky, A.V.Tur, Yu. N. Maslovsky, Zh. Eksper. Teor. Fiz., 106, 1, 187 (2008).

2. V.V.Yanovsky, A.V.Tur, N. Maslovsky, Theor. Mathem. Phys., 175, 655 (2013).

3. S.V.Slipushenko, A.V.Tur, V.V.Yanovsky, Zh. Eksper. Teor. Fiz., 117, 2, 274 (2013).

4. M.A.Ratner, A.V.Tur, V.V.Yanovsky, Functional Materials, 20, 4, 510 (2013).

5. G.A.Galperin, A.N.Zemlyakov, Mathematical Billiards, Nauka, Moscow, 1990 (in Russian).

6. S.Tabachnikov, Geometry and Billiards, American Mathematical Society, September 28, 2005, 176p.

7. Ya.G.Sinai, Entropy per Particle for the Dynamical System of Hard Spheres, Preprint, Harvard University, 1978.

8. B.A.Rozenfeld, Mnogomernye Prostranstva, Nauka, Moscow, 1966 (in Russian).

9. N.I.Chernov, Funct. Anal. Appl., 25, 204 (1991).

10. L.A.Bunimovich, Dispersing, Defocusing and Astigmatism, Math. Education 5, p.106-124, 2001(in Russian).

11. S.Lansel, M.A.Porter, L.A.Bunimovich, Chaos, 16, 013129 (2006). 